\title{
Combinatorial Influences of Paclitaxel and Strain on Axonal Transport
}

\author{
Brian G. Bober ${ }^{1}$, Edgar Gutierrez ${ }^{2}$, Steven Plaxe ${ }^{3}$, Alex Groisman ${ }^{2}$, Sameer B. Shah ${ }^{1,4^{*}}$ \\ ${ }^{1}$ Department of Bioengineering, University of California, San Diego, La Jolla, CA \\ ${ }^{2}$ Department of Physics, University of California, San Diego, La Jolla, CA \\ ${ }^{3}$ Department of Reproductive Medicine, University of California, San Diego, La Jolla, CA \\ ${ }^{4}$ Department of Orthopaedic Surgery, University of California, San Diego, La Jolla, CA
}

${ }^{*}$ Address correspondence to:

9500 Gilman Drive, MC 0863

La Jolla, CA 92093

(858) 822-0720 (phone)

(858) 822-3807 (fax)

sbshah@ucsd.edu

Keywords: paclitaxel, axonal transport, biomechanics, strain, mitochondria, actin, imaging, kymograph

Abstract: Paclitaxel is an effective chemotherapeutic that, despite its common use, often causes peripheral sensory neuropathy. In neurons, paclitaxel binds to and stabilizes microtubules, and through unknown mechanisms, bundles microtubules and disrupts their organization. Because microtubules serve as tracks on which a variety of axonal cargoes are transported, a leading hypothesis for the etiology of paclitaxel-induced neuropathy is that these changes to microtubule organization impair axonal transport. In addition to supporting transport, microtubules also serve a structural role, accommodating axonal extension occurring during axonal growth or joint movement. In light of this dual role for microtubules, we tested the hypothesis that axonal stretch amplified the effects of paclitaxel on axonal transport. Embryonic rat dorsal root ganglia were cultured on stretchable silicone substrates, and parameters describing the axonal transport of three distinct cargoes - mitochondria, synaptophysin, and actin - were measured with and without paclitaxel treatment and axonal strain. Paclitaxel treatment, particularly in combination with stretch, led to severe perturbations in a number of transport parameters, including the number, velocity, and travel distance of cargoes in the axon. Our results suggest that mechanical loading of neurons can exacerbate transport deficits associated with paclitaxel treatment, raising the interesting possibility that paclitaxel influences neuronal function in a multi-factorial manner. 


\section{Introduction}

Paclitaxel is a taxane used to treat a variety of metastatic cancers, including breast, ovarian, and lung cancer. While effective as an anti-cancer agent, paclitaxel causes peripheral sensory neuropathy in as many as $60 \%$ of treated patients ${ }^{1}$.

Symptoms are dose dependent and can include loss of sensory function, paresthesia, neuropathic pain, and myalgia. Peripheral neuropathy is generally cited as the dose limiting side effect of paclitaxel treatment ${ }^{2}$.

Mechanistically, paclitaxel binds to and stabilizes microtubules. One function of microtubules is to serve as tracks on which kinesin and dynein motor proteins shuttle cargoes along the length of the axon. Because paclitaxel affects the microtubule tracks and induces neuropathic symptoms in a stocking-and-glove distribution, progressing distally to proximally ${ }^{1}$, several studies have investigated impairment of axonal transport as a potential cause of neurotoxicity. Accordingly, it is well documented that paclitaxel slows down bulk axonal transport of a wide variety of cargoes, ranging from cytoskeletal proteins to vesicles and organelles ${ }^{3-6}$, presumably due to abnormal microtubule aggregation and organization ${ }^{7,8}$.

In addition to their pivotal roles in axonal transport, microtubules also provide structural support in the axonal shaft. In the peripheral nervous system, during phases of organismal growth and due to the movement of spanned joints, nerves undergo significant translation ${ }^{9}$ and incur considerable tensile strain $(\text { stretch })^{10}$. The cytoskeleton, including the microtubules, bears loads ${ }^{11}$ and is necessarily reorganized during axonal stretch ${ }^{12,13}$ ENREF 12 . Healthy neurons thus accommodate stretch without 
being damaged ${ }^{14}$. In fact, an in vitro study suggests that tension is necessary for the survival of neurons ${ }^{15}$. Paclitaxel alters the neuronal response to high strain-rate (traumatic) loading ${ }^{16}$ and increases the compressive modulus of neurons ${ }^{17}$ and thus may directly impact the ability of a neuron to tolerate deformation. A recent study indicates that deformation-induced mechanical damage can result from cytoskeletal perturbation; intriguingly, mutation of spectrin in a Caenorhabditis elegans model resulted in increased damage to axons lengthened during crawling ${ }^{18}$.

Recent studies have suggested that tensile strain can modify patterns of axonal transport. While theoretical and experimental evidence suggests that high strain rates fracture microtubules, thus resulting in transport failure ${ }^{19}$, lower, physiological levels of stretch are not necessarily detrimental for axonal transport. Specifically, mitochondrial velocity and transport frequency in stretch-grown axons appears unaffected for strains $<24 \%{ }^{20}$. In addition, Aplysia neurons display an increased likelihood of active transport of vesicles following stretch, with a retrograde bias ${ }^{21}$. Beyond these studies, though, despite mechanical and transport roles for neuronal microtubules, very little is known about the interaction between mechanical loading and axonal transport. Here we cultured cells on stretchable substrates integrated in novel microfabricated devices and used high-resolution confocal microscopy to examine the combined effect of stretching and paclitaxel treatment on axonal transport. Specifically, we tested whether paclitaxel impaired the axonal transport of three important axonal cargoes with different functions and transport dynamics - mitochondria, actin, and synatophysin-associated vesicles and whether axonal stretching amplified this effect. Our results revealed that axonal 
stretching and paclitaxel synergistically perturbed cargo transport, raising the interesting possibility that paclitaxel influences neuronal function in a multi-factorial manner. 


\section{Methods}

\section{Cell culture}

Dorsal root ganglia (DRG) were dissected from E15 embryonic rats as previously described $^{22}$. DRGs were digested in trypsin for 30 minutes, collected by centrifugation at 100x gravity, and pre-plated for 75 minutes to minimize the presence of non-neuronal cells. Cells were then recollected and cultured on a stretchable polydimethylsiloxane (PDMS) substrate at the bottom of a $1 \times 8 \mathrm{~mm}$ micro-cuvette integrated in a custom-built microfabricated device, which was also made out of PDMS. The PDMS substrate could be continuously extended by up to $17 \%$ (as assessed by displacements of beads bound to its surface) along the direction of its shorter $(1 \mathrm{~mm})$ dimension. (Technical details of the device are published elsewhere ${ }^{23}$.) Cells were grown in Minimum Essential Media supplemented with $10 \%$ FBS, $1 \%$ PSN, $50 \mathrm{ng} / \mathrm{mL}$ nerve growth factor, and $2 \%$ B27 at $37^{\circ} \mathrm{C}$ for $24-48$ hours before imaging.

\section{Reagents}

Paclitaxel (TCl, P1632) was dissolved in DMSO and stored at $-20^{\circ} \mathrm{C}$ as $10 \mathrm{mM}$ stocks. Cells treated with paclitaxel were incubated at $100 \mathrm{nM}$ paclitaxel in culture media for two hours prior to their imaging as well as during the course of the imaging. MitoTracker® Green FM dye (Invitrogen, Carslbad, CA), a fluorescent probe which localizes to mitochondria, was utilized according to manufacturer specifications to visualize mitochondrial transport in DRGs. CellLight@ Reagents BacMam 2.0 (Life Technologies), a baculovirus delivery system conferring expression of synaptophysinRFP and actin-GFP that has been confirmed to infect neurons ${ }^{24}$ (and was previously 
used to study actin flow ${ }^{25}$ ), was used to visualize synaptophysin and actin transport along DRG axons.

Imaging and analysis

After 24-48 hours of growth, PDMS devices were placed on a Leica SP5 confocal microscope within an environmental chamber (Tokai Hit) which maintained a temperature of $37 \stackrel{\circ}{\mathrm{C}}$ with constant airflow of $5 \% \mathrm{CO}_{2}$. The stretchable substrates were gradually extended at a relatively low strain rate of $\sim 0.5 \% / \mathrm{sec}$ (to prevent possible damage from fast extension) up to a maximal strain of $16.8 \pm .4 \%$. Cells selected for imaging on stretched substrates had axons aligned $\pm 20 \%$ with the axis of stretch and were imaged during the first 15 minutes post stretch.

DRGs were imaged using a 63x glycerol-water mixture immersion lens (NA = 1.3), and fluorescent images of the cargoes were taken along with either DIC or brightfield images of the axon. Movies of axonal transport were then assembled with frames taken every 5 seconds for 5 minutes for mitochondria and actin-GFP transport, and every 5 seconds for 90 seconds for synaptophysin-RFP transport. Kymographs were generated from movies using a custom MATLAB script to allow for visualization of mobile and stationary tracks and computation of velocity, movement time, and distance travelled (procedure adopted with modifications from ${ }^{26}$ ). All transport related parameters were calculated over the entire duration of each movie. Transport of actin in the growth cone was excluded from analysis. In the case of mitochondrial transport, only relatively fast moving cargoes were considered, with those traveling at velocities 
less than $.05 \mu \mathrm{m} / \mathrm{sec}$ ignored due to previously documented low velocity mitochondrial and cytoskeletal drift associated with growth cone induced tension ${ }^{27}$.

\section{Transport Parameters}

Parameters used to describe mitochondria and synaptophysin-RFP movement included cargo velocity, defined as the average velocity of the cargo while it was in motion, track time, defined as the time a cargo moved without pausing or changing directions, and distance travelled, defined as the distance a cargo moved before it paused or changed directions. Because of frequent pauses and direction changes observed in the kymographs of actin-GFP, to describe actin-GFP movement, we tracked the net displacement of actin-GFP densities during their imaging period. The data was then used to calculate the average velocity of actin during periods of movement. We also analyzed the total time that actin-GFP density was stationary or mobile during the imaging period. Additionally, based on the direction of motion, all resolvable cargoes were scored as anterograde, retrograde, or, in the case of mitochondria, stationary, in order to calculate percentages or numbers of mobile cargoes.

\section{Statistics}

Mean values were compared using two-way ANOVA, testing for main effects of paclitaxel treatment and stretch. Because sample sizes varied between groups, likely due to the treatments leading to lower numbers of observations per analysis period, and no significant interaction between main effects was detected in the parameters analyzed, type 2 sum of squares was used in all ANOVA calculations. Post-hoc testing 
between all groups was performed using the Tukey-Kramer method, which accounts for multiple comparisons. P-values less than 0.05 were considered statistically significant.

\section{$\underline{\text { Results }}$}

\section{Mitochondrial Transport}

To test the combinatorial influences of stretch and paclitaxel treatment on mitochondria transport, we measured a number of parameters; for convenience and clarity, we provided a comprehensive set of mean values of the parameters with significant ANOVA main effects noted (Table 1) as well as post-hoc p-values (Appendix Table A.1). Key results are presented below. Kymographs from each experimental group are shown (fig 2a). A representative movie of mitochondria axonal transport is also shown (supplementary movie 1).

As a first step towards determining the effects of stretch and paclitaxel treatment, we quantified the number and proportion of mitochondria moving anterogradely, retrogradely, or remaining stationary during the imaging period (5 minutes). Under control conditions, axons showed an average of about $60 \pm 21 \%$ stationary mitochondria, while the remaining mitochondria population equally experienced anterograde or retrograde movement, albeit with large variability from axon to axon (fig 2b). Two-way ANOVA showed significant effects of paclitaxel treatment $(p<.005)$ and stretch $(p<.05)$ on the proportion of moving mitochondria. Post-hoc analysis revealed that paclitaxel alone significantly increased the proportion of stationary mitochondria compared to control $(p<.01)$ by suppressing movement bidirectionally $(p<0.10$ for each direction). Stretch alone also trended towards increased proportions of stationary 
mitochondria, although to a lesser degree than paclitaxel $(p<.10)$. The combination of both stretch and paclitaxel treatment led to the most consistent and largest increase in stationary mitochondria $(p<.0005)$, driven by significant reductions in both anterograde $(p<.05)$ and retrograde $(p<.05)$ transport frequencies.

We next examined the directional transport capabilities of mitochondria that did move (i.e., were non-stationary) (fig $2 c-e$ ). Parameters used to describe cargo movement included cargo velocity, track time, and distance travelled, as defined in the Methods section. Two-way ANOVA revealed a significant effect of paclitaxel treatment on bidirectional velocity $(p<.005)$ and distance travelled $(p<.05)$, and a significant effect of stretch on anterograde track time $(p<.05)$ and distance traveled. Post-hoc analysis largely confirmed ANOVA trends, but additionally further elucidated possible combinatorial effects of paclitaxel and stretch treatment. Paclitaxel treatment alone induced strongly trending, though non-significant, decreases in mean anterograde distance travelled $(p=.08)$, retrograde distance travelled $(p=.13)$ and retrograde velocity $(p=.10)$. Anterograde velocity appeared lower, however, differences were not significant due to high variability in this parameter across axons $(p=.33)$. Stretch alone showed no significant effect on velocity, though it did reduce the anterograde distance travelled $(p<.05)$ by decreasing track time (indicated by ANOVA, though no post-hoc significance was detected due to large data variability).The combination of paclitaxel and stretch led to the largest and most significant reductions in velocity $(\mathrm{p}<.05$ for anterograde compared to stretch alone and retrograde compared to control and stretch treated neurons) and anterograde distance travelled $(p<.01)$, highlighting the inability of paclitaxel treated axons to accommodate stretch. 


\section{Synaptophysin-RFP Transport}

To test the effects of paclitaxel and axonal stretch on a cargo that undergoes fast transport, a similar analysis was performed on DRGs expressing synaptophysin-RFP. A comprehensive set of mean values of the parameters with significant ANOVA main effects noted (Table 2) as well as post-hoc p-values (Appendix Table A.2) are provided. Kymographs from each experimental group are shown (fig 3a). A representative movie of synaptophysin-RFP axonal transport is also shown (supplementary movie 2).

Similar transport distributions compared to mitochondria were observed in the transport of synaptophysin-RFP (fig 3b). Because synaptophysin-RFP particles appeared dimmer and smaller than labelled mitochondria, the total number of discrete particles in an axon could not be reliably resolved. We therefore analyzed the transport of synaptophysin-RFP in terms of raw numbers of mobile particles captured during the imaging period rather than proportions moving in each direction (fig 3b). Two-way ANOVA showed a significant effect of paclitaxel, but not stretch, on the number of mobile synaptophysin-RFP particles $(\mathrm{p}<.05)$. Post-hoc testing further revealed a significant reduction in the number of mobile anterograde particles only following the combination of paclitaxel and stretch $(p<.05)$, indicating that, as for mitochondria, combining these two treatments leads to the most severe transport perturbation. Both paclitaxel alone and paclitaxel treatment in combination with stretch led to similar, though not quite significant, reductions in retrograde transport frequency $(p<10)$.

We next examined the directional transport capabilities of synaptophysin-RFP particles that were mobile (fig $3 c-e$ ). Two-way ANOVA revealed a significant effect of 
paclitaxel treatment on bidirectional velocity $(p<.001)$ and distance travelled $(p<.01)$ and a significant effect of stretch on anterograde track time $(p<.05)$, velocity $(p<.01)$, and distance travelled $(p<.01)$. Post-hoc analysis showed that paclitaxel treatment alone led to a significant decrease in the bidirectional velocity $(p<.05)$ and trended towards a reduction in anterograde distance travelled $(p<10)$ of synaptophysin-RFP. Stretch alone showed no significant effect on velocity, though it did trend towards reduction in the anterograde distance travelled $(p<.07)$. Again, the combination of paclitaxel and stretch led to the most significant reductions in velocity $(p<.0001$ anterograde, $p<.005$ retrograde) and anterograde distance travelled $(p<.005)$. In addition, a significant decrease in anterograde velocity was observed in neurons treated with paclitaxel and stretch as compared to neurons treated with only paclitaxel $(p<.05)$, further indicating a combinatorial effect of the two treatments.

Taken together, these data reveal that paclitaxel reduces the velocity and frequency at which both mitochondria and synaptophysin-RFP move, while stretch tends to marginally reduce only the duration of a bout of movement. In combination, however, the two treatments led to an extremely large velocity reduction, causing the largest reduction in distance travelled by mobile particles. Additionally, the least number of mobile particles are observed in neurons treated with both paclitaxel and stretch, further implying that the two treatments synergistically perturb axonal transport.

\section{Actin Transport}

We next examined the transport of actin, because we posited that the transport of cytoskeletal cargoes may be particularly responsive to mechanical loading and 
changes in cytoskeletal stability. Mean values as well as $p$-values of different parameters from ANOVA and post-hoc significance testing are summarized in Table 3 and Appendix Table A.3. Representative dynamics of fluorescently labeled actin along the axon are shown in supplementary movie 3.

We focused our analysis on the movement of mobile actin-GFP densities appearing brighter than the ubiquitous actin background (fig 4a), which we have identified to be predominantly filamentous (data not shown). Actin transport dynamics were more complex than those of mitochondrial transport, as several densities appeared and disappeared during the imaging window, presumably due to polymerization and depolymerization, respectively. There were also numerous stationary patches of actin, which had varying intensity. Thus, as for synaptophysinRFP, we measured and compared raw numbers of moving actin densities ( 5 minute imaging period).

We first analyzed changes in the number and directionality of movement of actin densities in response to paclitaxel treatment and/or stretch (fig $4 b$ ). Paclitaxel treatment caused a significant decrease in the number of anterograde, but not retrograde, densities $(p<.05)$. While stretch alone did not change the number of densities moving in either direction, the combination of paclitaxel and stretch caused a massive reduction in the number of anterograde densities $(p<.005)$, with densities in only 2 out of 11 axons showing any anterograde movement. ANOVA showed a significant effect of paclitaxel on the number of mobile densities bidirectionally $(p<.005)$. 
As we did for mitochondria and synaptophysin-RFP, we next analyzed changes in the movement characteristics of individual mobile densities. We tracked actin density velocities, net displacements, and directional track times, as defined in the Methods section. Net displacements across all groups were highly variable (Table 3). While net displacement trended downward in response to paclitaxel treatment, no significant changes were detected by ANOVA or post-hoc testing. There were no significant differences in anterograde or retrograde track time among any groups.

We also compared directional actin density velocities (fig $4 c$ ). The movement of actin densities was nearly an order of magnitude slower than mitochondrial or synaptophysin-RFP transport. ANOVA revealed a significant effect of paclitaxel treatment on actin velocity in both directions $(p<.005)$. Post-hoc analysis showed paclitaxel treatment alone caused a significant decrease in the retrograde $(p<.05)$ velocity of moving actin densities and a strong trend towards a decrease in the anterograde velocity $(p=.08)$. Stretch alone did not yield significantly different velocity than in control data; in fact, anterograde actin density velocity was significantly higher in stretched neurons than in neurons treated with paclitaxel or paclitaxel in combination with stretch (bidirectionally, $p<.05$ ). The combination of stretch and paclitaxel treatment did not cause any significant difference in the velocity of moving actin densities compared to control, though there were few anterogradely moving densities detected at all, potentially rendering this comparison unreliable.

\section{Discussion}


Based on the unique dual role of microtubules, which both contribute to axonal structure and facilitating transport, this study examined the combinatorial effects of paclitaxel and axonal stretch on axonal transport dynamics of cargoes with distinct transport and functional profiles: mitochondria, synaptophysin, and actin (Tables 1,2 and 3). Paclitaxel reduced transport of all cargoes by most measures, except for duration of a bout of movement (fig 2-4). Stretch decreased the duration of movement (fig 2,3 e) and distance travelled (fig 2,3 c) by mitochondria and synaptophysin, but not their velocity (fig 2,3 d). However, stretch had minimal effects on the movement of actin densities (fig 4), confirming the capacity for the cytoskeleton to accommodate deformation. Compellingly, the combination of paclitaxel and stretch severely impaired the movement of all cargoes, showing the largest impairment of bidirectional mitochondrial and synaptophysin transport and of anterograde transport of actin.

Effects of paclitaxel and mechanical loading on mitochondrial and synaptophysin transport

Mitochondrial transport has been previously studied in both untreated neurons $s^{27,28}$ and paclitaxel treated neurons $s^{6,29-31}$. Indirect evidence from electron micrograph cross sections of nerves bathed in paclitaxel indicates buildup of organelles $^{29}$, including mitochondria ${ }^{30}$, suggesting transport impairment. A recent study in rat cortical neurons more directly showed depression of velocities and run lengths after paclitaxel treatment ${ }^{31}$, but did not examine changes in the proportion of moving mitochondria. Finally, a single study examined the effect of axonal strain on mitochondrial transport frequency and velocity ${ }^{20}$, but revealed impaired transport frequency only at extremely large strains. The data presented in our study agree with 
much of the previous literature in that paclitaxel treatment reduces mitochondrial velocity and distance travelled, while stretch does not affect mitochondrial velocity or the proportion of transported mitochondria. Also, our control measurements of mitochondrial velocity and distances travelled are similar in magnitude to previously reported literature values, supporting the validity of our quantification methods ${ }^{31}$.

Our measured velocities of synaptophysin transport in untreated axons ranged from $0.13 \mu \mathrm{m} / \mathrm{sec}$ to $1.63 \mu \mathrm{m} / \mathrm{sec}$, which were on the same order of magnitude, but slightly lower on average than the published data ${ }^{32}$. These differences may be due to the fact that prior studies examined synaptophysin transport in networked central neurons rather than DRG axons ${ }^{32,33}$. In addition, our use of a lower frame rate than in prior studies and inclusion of lower velocity movements in reported averages may have contributed to the lower reported mean velocity.

In our study, stretch alone minimally altered transport; however, in combination with paclitaxel, contributed to substantial transport impairment. Thus, paclitaxel, which changes the structure and organization of the axonal cytoskeleton, fundamentally alters the ability of a neuron to respond to mechanical loading. Mechanisms by which this occurred are not clear, however, previous research on traumatic axonal stretch injury provides possible clues. Traumatic stretch has been implicated in microtubule breakage and alterations in axonal morphology, and the structural damage is increased in its extent and persists longer in paclitaxel treated neurons ${ }^{16,34}$. In particular, traumatic stretch injury to cultured neurons at high strain ( 30\%) and high strain rates caused microtubule breakage, modified the ability of axons to restore tension after unloading, and caused regional bulging suggestive of transport impairment. Thus, microtubule 
breakage, albeit at a lower level due to the more physiological levels of strain and lower strain rates, may partially explain our observations of decreases in distance traveled and movement duration, in the absence of any changes in velocity.

\section{Effects of paclitaxel and mechanical loading on actin transport}

Radiolabeling experiments have indicated that bulk transport of actin may be impaired by paclitaxel treatment ${ }^{4}$, but, to our knowledge, the specific processes contributing to this impairment have not yet been analyzed. Moreover, mechanisms of the axonal transport of actin remain largely undescribed, with most studies either focusing on subsets of movement ${ }^{35,36}$ or indirect measurements of general cytoskeletal mobility ${ }^{14,20,37}$ that have not yet revealed the complex mechanisms of actin transport as a whole. We note that it is well documented that axonal stretch positively regulates axonal growth rate without a reduction in axonal diameter ${ }^{14,20,38}$ and accelerated growth of axonal volume should lead to increased demand for structural proteins. Nevertheless, the potential role of stretch in the production and/or transport of cytoskeletal proteins in neurons remains unclear.

In this work, we have begun to address these gaps of knowledge by examining the movement of bright actin densities along the axon in response to axonal stretch and/or paclitaxel treatment. Interestingly, paclitaxel showed a number of deleterious effects on actin transport, suggesting a role for microtubules in the transport or inhibition of transport of actin. Compared to the non-cytoskeletal cargoes analyzed, stretch alone had little effects on actin transport. The exact reason for these differences is unknown, but could be related to the comparatively smaller distances and slower velocities at 
which actin appeared to move, or differences in the response of load-bearing cargoes (e.g., cytoskeletal elements such as actin) versus non-load bearing cargoes (e.g., organelles) to stretch. However, when paclitaxel treatment and stretch were combined, there was a significant reduction in anterograde actin density movement, again suggesting that paclitaxel treatment leads to deterioration in the ability of neurons to properly adapt to stretch.

\section{Conclusions and future directions}

Several conclusions may be drawn from our findings that stretch and paclitaxel treatment have a synergistic effect on impairing axonal transport. Because paclitaxel treatment led to less frequent bouts of movement of all cargoes analyzed, the drug likely decreased the number of microtubule tracks in an axon capable of supporting transport. Paclitaxel additionally decreased the velocity at which each cargo travelled, indicating altered interactions between motor proteins and microtubules. Alternately, previously observed microtubule misalignment ${ }^{6}$ or bundling could lead to slower net movement along the principal axonal vector due to interference with transported cargoes. Axonal stretch typically led to shorter track times, which, in light of previous research, may be indicative of breakage or separation of microtubule tracks, thus leading to shorter bursts of movement for fast moving cargoes. In contrast, actin density transport showed little changes in response to stretch, suggesting that actin transport is regulated differently than the transport of faster moving, non-cytoskeletal cargoes. The combination of both stretch and paclitaxel treatment often led to the largest transport deficits across all cargoes - less functional tracks, shorter tracks, and lower transport velocities are all detrimental to axonal transport. 
Our work fills some gaps in the literature by detailing a wide array of effects of paclitaxel treatment and stretching on transport of various cargoes. Most importantly, however, our results indicate that paclitaxel treatment, which changes the structure and organization of the axonal cytoskeleton, fundamentally alters the ability of a neuron to respond to axonal loading. While caution must be used in extrapolating in vitro studies to in vivo environment, our results raise the currently untested possibility that axonal stretch, such as that induced by joint motion ${ }^{9,39,40}$, could exacerbate transport defects associated with paclitaxel-induced neuropathy. Testing this hypothesis provides a compelling direction for future study.

\section{Acknowledgements}

We gratefully acknowledge funding support from the National Science Foundation (CBET-0932590 and CMMI-1130997, S.B.S.), National Institute of Health (GM098412, E.G. and A.G), the National Skeletal Muscle Research Center at UCSD (S.B.S.), and the UCSD Academic Senate (S.B.S.). We appreciate valuable discussions with Dr. Velia Fowler, Dr. Richard Lieber, the Neuromuscular Bioengineering Laboratory and the Muscle Physiology Laboratory.

\section{References}

1. Argyriou AA, Kyritsis AP, Makatsoris T, Kalofonos HP. Chemotherapy-induced peripheral neuropathy in adults: a comprehensive update of the literature. Cancer Manag Res 2014;6:135-47. 2. Scripture CD, Figg WD, Sparreboom A. Peripheral neuropathy induced by paclitaxel: recent insights and future perspectives. Curr Neuropharmacol 2006;4:165-72.

3. Lapointe NE, Morfini G, Brady ST, Feinstein SC, Wilson L, Jordan MA. Effects of eribulin, vincristine, paclitaxel and ixabepilone on fast axonal transport and kinesin-1 driven microtubule gliding: Implications for chemotherapy-induced peripheral neuropathy. Neurotoxicology 2013;37:231-9. 4. Komiya $Y$, Tashiro T. Effects of taxol on slow and fast axonal transport. Cell Motil Cytoskeleton 1988;11:151-6. 
5. Theiss C, Meller K. Taxol impairs anterograde axonal transport of microinjected horseradish peroxidase in dorsal root ganglia neurons in vitro. Cell Tissue Res 2000;299:213-24.

6. Shemesh OA, Spira ME. Paclitaxel induces axonal microtubules polar reconfiguration and impaired organelle transport: implications for the pathogenesis of paclitaxel-induced polyneuropathy. Acta Neuropathol 2010;119:235-48.

7. Turner PF, Margolis RL. Taxol-induced bundling of brain-derived microtubules. J Cell Biol 1984;99:940-6.

8. Masurovsky EB, Peterson ER, Crain SM, Horwitz SB. Microtubule arrays in taxol-treated mouse dorsal root ganglion-spinal cord cultures. Brain Res 1981;217:392-8.

9. Topp KS, Boyd BS. Structure and biomechanics of peripheral nerves: nerve responses to physical stresses and implications for physical therapist practice. Phys Ther 2006;86:92-109.

10. Aoki M, Takasaki H, Muraki T, Uchiyama E, Murakami G, Yamashita T. Strain on the ulnar nerve at the elbow and wrist during throwing motion. J Bone Joint Surg Am 2005;87:2508-14.

11. Ouyang $\mathrm{H}, \mathrm{Nauman} \mathrm{E}$, Shi R. Contribution of cytoskeletal elements to the axonal mechanical properties. J Biol Eng 2013;7:21.

12. Chetta J, Kye C, Shah SB. Cytoskeletal dynamics in response to tensile loading of mammalian axons. Cytoskeleton (Hoboken) 2010;67:650-65.

13. Peter SJ, Mofrad MR. Computational modeling of axonal microtubule bundles under tension. Biophys J 2012;102:749-57.

14. Pfister BJ, Iwata A, Meaney DF, Smith DH. Extreme stretch growth of integrated axons. J Neurosci 2004;24:7978-83.

15. Anava S, Greenbaum A, Ben Jacob E, Hanein $Y$, Ayali A. The regulative role of neurite mechanical tension in network development. Biophys J 2009;96:1661-70.

16. Tang-Schomer MD, Johnson VE, Baas PW, Stewart W, Smith DH. Partial interruption of axonal transport due to microtubule breakage accounts for the formation of periodic varicosities after traumatic axonal injury. Exp Neurol 2012;233:364-72.

17. Au NP, Fang Y, Xi N, Lai KW, Ma CH. Probing for chemotherapy-induced peripheral neuropathy in live dorsal root ganglion neurons with atomic force microscopy. Nanomedicine 2014.

18. Hammarlund M, Jorgensen EM, Bastiani MJ. Axons break in animals lacking beta-spectrin. J Cell Biol 2007;176:269-75.

19. Ahmadzadeh H, Smith DH, Shenoy VB. Viscoelasticity of tau proteins leads to strain ratedependent breaking of microtubules during axonal stretch injury: predictions from a mathematical model. Biophys J 2014;106:1123-33.

20. Loverde JR, Ozoka VC, Aquino R, Lin L, Pfister BJ. Live imaging of axon stretch growth in embryonic and adult neurons. J Neurotrauma 2011;28:2389-403.

21. Ahmed WW, Saif TA. Active transport of vesicles in neurons is modulated by mechanical tension. Sci Rep 2014;4:4481.

22. Li R. Culture methods for selective growth of normal rat and human Schwann cells. Methods Cell Biol 1998;57:167-86.

23. E. Gutierrez BB, E.V. Tkachenko, S.B. Shah, A. Groisman. Micro-fabricated devices with stretchable silicone substrates and micro-patterned silicone gels for experiments on adherent cells with high-resolution imaging. Mol Biol Cell2014:415.

24. Boyce FM, Bucher NL. Baculovirus-mediated gene transfer into mammalian cells. Proc Natl Acad Sci U S A 1996;93:2348-52.

25. Koch D, Rosoff WJ, Jiang J, Geller HM, Urbach JS. Strength in the periphery: growth cone biomechanics and substrate rigidity response in peripheral and central nervous system neurons. Biophys J 2012;102:452-60. 
26. Love JM, Pathak GK, Chetta J, Shah SB. Variability in Membrane Continuity Between Schwann Cells and Neurons. Cellular and Molecular Bioengineering 2012;5:450-62.

27. Miller KE, Sheetz MP. Direct evidence for coherent low velocity axonal transport of mitochondria. J Cell Biol 2006;173:373-81.

28. Saxton WM, Hollenbeck PJ. The axonal transport of mitochondria. J Cell Sci 2012;125:2095-104.

29. Nakata T, Yorifuji H. Morphological evidence of the inhibitory effect of taxol on the fast axonal transport. Neurosci Res 1999;35:113-22.

30. Roytta M, Horwitz SB, Raine CS. Taxol-induced neuropathy: short-term effects of local injection. J Neurocytol 1984;13:685-701.

31. Das V, Sim DA, Miller JH. Effect of taxoid and nontaxoid site microtubule-stabilizing agents on axonal transport of mitochondria in untransfected and ECFP-htau40-transfected rat cortical neurons in culture. J Neurosci Res 2014.

32. Roy S, Winton MJ, Black MM, Trojanowski JQ, Lee VM. Rapid and intermittent cotransport of slow component-b proteins. J Neurosci 2007;27:3131-8.

33. Welzel O, Knorr J, Stroebel AM, Kornhuber J, Groemer TW. A fast and robust method for automated analysis of axonal transport. Eur Biophys J 2011;40:1061-9.

34. Tang-Schomer MD, Patel AR, Baas PW, Smith DH. Mechanical breaking of microtubules in axons during dynamic stretch injury underlies delayed elasticity, microtubule disassembly, and axon degeneration. FASEB J 2010;24:1401-10.

35. Ruthel G, Banker G. Actin-dependent anterograde movement of growth-cone-like structures along growing hippocampal axons: a novel form of axonal transport? Cell Motil Cytoskeleton 1998;40:160-73.

36. Flynn KC, Pak CW, Shaw AE, Bradke F, Bamburg JR. Growth cone-like waves transport actin and promote axonogenesis and neurite branching. Dev Neurobiol 2009;69:761-79.

37. Chada SR, Hollenbeck PJ. Nerve growth factor signaling regulates motility and docking of axonal mitochondria. Curr Biol 2004;14:1272-6.

38. Zheng J, Lamoureux P, Santiago V, Dennerll T, Buxbaum RE, Heidemann SR. Tensile regulation of axonal elongation and initiation. J Neurosci 1991;11:1117-25.

39. Mahan MA, Vaz KM, Weingarten D, Brown JM, Shah SB. Altered Ulnar Nerve Kinematic Behavior in a Cadaver Model of Entrapment. Neurosurgery 2015.

40. Phillips JB, Smit X, De Zoysa N, Afoke A, Brown RA. Peripheral nerves in the rat exhibit localized heterogeneity of tensile properties during limb movement. J Physiol 2004;557:879-87. 


\section{Figure legends}

Figure 1 - Dorsal root ganglia cells can be cultured and stretched on flexible substrates

a) Beads attached to surface of stretch device before (red) and after (green) stretch. After stretch, pairs of beads attached to the surface appear further apart from one another, allowing for the calculation of strain. b) Dorsal root ganglia neurons cultured on the stretchable substrate and treated with MitoTracker to show fluorescent mitochondria. Axons are oriented randomly, so only those along the axis of stretch (dashed arrow) \pm 20 degrees are sampled and analyzed for the effect of stretching on axonal transport. c) Bundle of axons originating from cell bodies located at the left side of the image (*) at rest (top) and immediately after release from stretch (bottom). The axons show undulations (highlighted by arrows) after releasing stretch, indicating that axons remain adherent to the substrate during stretch. Scale bar $=25 \mu \mathrm{m}$. D) Unstretched (top) and stretched (bottom) neuron, note the elongation of the cell body (*) post-stretch. Scale bar $=25 \mu \mathrm{m}$.

Figure 2 - Mitochondrial transport observed with and without paclitaxel treatment and stretch in cultured rat dorsal root ganglia

a) Kymographs of mitochondrial transport observed over 5 minutes in axons, from left to right, with no treatment, stretch treatment alone, paclitaxel treatment alone, and paclitaxel treatment in combination with stretch. b) Graphs summarizing the directionality of mitochondrial transport over 5 minute viewing windows under each experimental condition. Paclitaxel treatment causes a significant increase in the proportion of stationary mitochondria; neurons treated with paclitaxel and stretch show the most consistant increase in stationary mitochondria, driven by significant reductions in bidirectional movement. c) Graphs of distances travelled by mitochondria under each experimental condition during 5 minutes of imaging. Stretch and paclitaxel treatment negatively disrupt mitochondria travel distance, with the combination of the two leading to the most consistent decreases in anterograde transport. d) Graphs of velocities of mitochondria under each experimental condition observed during 5 minutes of imaging. Stretch alone has no apparent effect on velocity, while paclitaxel treatment, particularly in combination with stretch, causes large decreases in the velocity of mobile mitochondria. e) Graphs of average duration of a bout of mitochondria movement under each experimental condition observed during 5 minutes of imaging. ANOVA reveals a significant main effect of stretch on anterograde track time, but no post-hoc significance between groups was detected. All error bars represent standard error of the mean. Bars on the graph indicate groups which show a significant $(\mathrm{p}<.05)$ difference from each other, as determined by Tukey's HSD. * indicates significant $(\mathrm{p}<.05)$ main effects of paclitaxel (P) and stretch (S) from two way ANOVA. Each experimental group contains 7-15 axons with 15-50 mobile tracks observed.

Figure 3 - Synaptophysin-RFP transport observed with and without paclitaxel treatment and stretch in cultured rat dorsal root ganglia

a) Kymographs of synaptophysin-RFP transport over 90 seconds in axons, from left to right, with no treatment, stretch treatment alone, paclitaxel treatment alone, and paclitaxel treatment in combination with stretch. b) Graphs of the transport of synaptophysin-RFP over 90 second viewing windows. Neurons treated with paclitaxel in combination with stretch show the least number of detectable mobile particles in the anterograde direction. c) Graphs of distances 
travelled by synaptophysin-RFP during 90 seconds of imaging. The combination of paclitaxel and stretch leads to the most significant reductions in distance travelled. d) Graphs of velocities of synaptophysin-RFP observed during 90 seconds of imaging. Paclitaxel causes a significant reduction in transport velocity, which is magnified by axonal stretch. In the anterograde direction, paclitaxel alone and paclitaxel + stretch groups show signifantly different velocities. e) Graphs of average duration of a bout of synaptophysin-RFP movement observed during 90 seconds of imaging. ANOVA reveals a main effect of stretch in the anterograde direction and paclitaxel in the retrograde direction, but little post-hoc significance was found. Bars on the graph indicate groups which show a significant $(\mathrm{p}<.05)$ difference from each other, as determined by Tukey's HSD. * indicates significant $(\mathrm{p}<.05)$ main effects of paclitaxel $(\mathrm{P})$ and stretch (S) from two way ANOVA and \# indicats a main effect of just outside of statistical significance $(\mathrm{p}=.08)$. Each experimental group contains $16-20$ axons with $40-80$ mobile densities observed.

Figure 4 - Actin-GFP transport observed with and without paclitaxel treatment and stretch in cultured rat dorsal root ganglia

a) Kymograph of actin-GFP transport over 5 minutes in axons, from left to right, with no treatment, stretch treatment alone, paclitaxel treatment alone, and paclitaxel treatment in combination with stretch. (b) Transport distribution of actin densities over 5 minute viewing windows. Paclitaxel treatment causes a reduction in the number of mobile actin densities. Stretch alone has little effect on this parameter. In combination with stretch, very few axons (2 out of 11) showed anterograde movement of actin densities. c) Moving velocity of actin densities observed during 5 minutes of imaging. Paclitaxel treatment causes a reduction in the velocity of mobile actin densities. Stretch alone has little or potentially small positive effects on this parameter. All error bars represent standard error of the mean. Bars on the graph indicate groups which show a significant $(\mathrm{p}<.05)$ difference from each other, as determined by Tukey's HSD. * indicates significant $(\mathrm{p}<.05)$ main effects of paclitaxel $(\mathrm{P})$ and stretch $(\mathrm{S})$ from two way ANOVA. Each experimental group contains 11-17 axons with 15-54 mobile tracks observed. 


\section{Table legends}

Table 1 - Mitochondiral transport parameters

Mitochondrial transport parameters presented as means \pm standard deviations. Significant main effects of Paclitaxel $(\mathrm{P})$ and Stretch $(\mathrm{S})(\mathrm{p}<.05)$ from ANOVA testing are indicated by * next to the category. Each experimental group contains 7-15 axons with 15-50 mobile tracks observed.

Table 2 - Synaptophysin transport parameters

Synaptophysin transport parameters presented as means \pm standard deviations. Significant main effects of Paclitaxel $(\mathrm{P})$ and Stretch $(\mathrm{S})(\mathrm{p}<.05)$ from ANOVA testing are indicated by * next to the category. Each experimental group contains 16-20 axons with 40-80 mobile densities observed.

Table 3 - Actin transport parameters

Actin transport parameters presented as means \pm standard deviations. Significant main effects of Paclitaxel $(\mathrm{P})$ and Stretch $(\mathrm{S})(\mathrm{p}<.05)$ from ANOVA testing are indicated by * next to the category. Each experimental group contains 11-17 axons with 15-54 mobile tracks observed. 


\section{Mitochondria Parameters (Mean $\underline{ \pm}$ St. Dev)}

\section{- Paclitaxel}

\section{Parameter}

Velocity (anterograde, $\mu \mathrm{m} / \mathrm{sec}$ ) P:*

Velocity (retrograde, $\mu \mathrm{m} / \mathrm{sec}$ ) P:*

Total distance (anterograde, $\mu \mathrm{m}$ ) P,S:* $13.800 \pm 8.546$

Total distance (retrograde, $\mu \mathrm{m}) \mathrm{P}$ * $^{*}$

Percent stationary tracks $\mathrm{P}, \mathrm{S}: *$

Percent anterograde tracks P:*

Percent retrograde tracks $\mathrm{P}, \mathrm{S}: *$

Track time (anterograde, sec) S:*

Track time (retrograde, sec)

\section{- Stretch}

$0.301 \pm 0.127$

$-0.373 \pm 0.188$

$-17.068 \pm 16.307$

$60.306 \pm 21.472$

$17.238 \pm 16.683$

$23.074 \pm 16.614$

$51.538 \pm 33.698$

$48.571 \pm 36.753$
+ Stretch

$0.324 \pm 0.205$

$-0.375+0.220$

$9.189 \pm 6.057$

$-10.711 \pm 8.921$

$72.794 \pm 13.178$

$14.552+7.862$

$12.654 \pm 10.369$

$36.167 \pm 29.175$

$32.115+19.502$
+ Paclitaxel

- Stretch

$0.222 \pm 0.115$

$0.154 \pm 0.057$

$-0.235 \pm 0.186$

$-0.165 \pm 0.086$

$8.134 \pm 4.237$

$5.116 \pm 2.995$

$-8.587 \pm 7.808 \quad-8.609 \pm 7.527$

$79.975 \pm 13.671$

$86.309 \pm 11.337$

$7.550 \pm 8.183$

$3.980 \pm 4.743$

$12.474 \pm 11.277$

$9.711 \pm 10.768$

$55.385 \pm 38.539$

$33.750 \pm 17.061$

$39.643 \pm 16.108$

$56.250+32.272$

Table 1 - Mitochondiral transport parameters

Mitochondrial transport parameters presented as means \pm standard deviations. Significant main effects of Paclitaxel $(\mathrm{P})$ and Stretch $(\mathrm{S})(\mathrm{p}<.05)$ from ANOVA testing are indicated by * next to the category. Each experimental group contains 7-15 axons with 15-50 mobile tracks observed. 


\section{Synaptophysin-RFP Parameters (Mean \pm St. Dev)}

\begin{tabular}{|c|c|c|c|c|}
\hline \multirow[b]{2}{*}{ Parameter } & \multicolumn{2}{|c|}{ - Paclitaxel } & \multicolumn{2}{|c|}{ + Paclitaxel } \\
\hline & - Stretch & + Stretch & - Stretch & + Stretch \\
\hline Velocity (anterograde, $\mu \mathrm{m} / \mathrm{sec}$ ) P,S:* & $0.498 \pm 0.303$ & $0.430 \pm 0.230$ & $0.307 \pm 0.202$ & $0.145 \pm 0.069$ \\
\hline Velocity (retrograde, $\mu \mathrm{m} / \mathrm{sec}$ ) P:* & $-0.441 \pm 0.297$ & $-.483 \pm .334$ & $-0.260 \pm 0.095$ & $-0.210 \pm 0.128$ \\
\hline Total distance (anterograde, $\mu \mathrm{m}$ ) P,S:* & $10.348 \pm 11.028$ & $5.878 \pm 4.733$ & $6.490 \pm 6.326$ & $2.862 \pm 2.398$ \\
\hline Total distance (retrograde, $\mu \mathrm{m}) \mathrm{P}: *$ & $-8.388 \pm 8.859$ & $-9.881 \pm 15.152$ & $-2.951 \pm 2.000$ & $-3.034 \pm 3.287$ \\
\hline Number anterograde tracks P:* & $1.667 \pm 1.017$ & $1.421 \pm 0.961$ & $1.316 \pm 0.946$ & $0.824 \pm 0.636$ \\
\hline Number retrograde tracks P:* & $1.667 \pm 1.528$ & $1.632 \pm 1.739$ & $0.579 \pm 0.902$ & $0.765 \pm 0.664$ \\
\hline Track time (anterograde, sec) S:* & $18.638 \pm 11.840$ & $13.759 \pm 8.622$ & $22.255 \pm 14.997$ & $18.185 \pm 9.228$ \\
\hline Track time (retrograde, sec) P:* & $18.674 \pm 12.770$ & $17.843+11.926$ & $11.621 \pm 6.746$ & $14.978+8.275$ \\
\hline
\end{tabular}

Table 2 - Synaptophysin transport parameters

Synaptophysin transport parameters presented as means \pm standard deviations. Significant main effects of Paclitaxel $(\mathrm{P})$ and Stretch $(\mathrm{S})(\mathrm{p}<.05)$ from ANOVA testing are indicated by * next to the category. Each experimental group contains 16-20 axons with 40-80 mobile densities observed. 


\section{Actin-GFP Parameters $($ Mean \pm St. Dev)}

- Paclitaxel

Parameter

Velocity (anterograde, $\mu \mathrm{m} / \mathrm{sec}$ ) P:*

Velocity (retrograde, $\mu \mathrm{m} / \mathrm{sec}$ ) $\mathrm{P}:^{*} \quad-0.075 \pm 0.055$

Net Displacement (anterograde, $\mu \mathrm{m}$ )

Net Displacement (retrograde, $\mu \mathrm{m})$

Total number mobile tracks $\mathrm{P}: *$

Total number anterograde tracks $\mathrm{P}:^{*}$

Total number retrograde tracks $\mathrm{P}$ :*

Track time (anterograde, sec)

Track time (retrograde, sec)
- Stretch

$0.069 \pm 0.050$

$2.583 \pm 2.284$

$-3.180 \pm 3.287$

$3.00+2.318$

$1.529 \pm 1.125$

$1.588 \pm 1.372$

$32.586 \pm 29.629$

$35.376 \pm 34.139$
+ Stretch

$0.093 \pm 0.051$

$-0.071 \pm 0.059$

$4.098 \pm 3.293$

$-2.828 \pm-2.925$

$3.727 \pm 1.794$

$1.364 \pm 0.809$

$2.364 \pm 1.690$

$28.222 \pm 35.212$

$43.856 \pm 42.065$
+ Paclitaxel

- Stretch

$0.032+0.023$

$-0.030 \pm 0.019$

$-0.052 \pm 0.038$

$1.954 \pm 1.801$

$3.403 \pm 3.141$

$-2.407 \pm 2.561$

$1.182 \pm 0.982$

$0.182 \pm 0.405$

$0.909 \pm 0.831$

$0.813 \pm 0.750$

$34.582 \pm 36.035$

$28.625 \pm 47.917$

$53.391 \pm 54.346 \quad 41.541+29.577$

Table 3 - Actin transport parameters

Actin transport parameters presented as means \pm standard deviations. Significant main effects of Paclitaxel (P) and Stretch $(\mathrm{S})(\mathrm{p}<.05)$ from ANOVA testing are indicated by * next to the category. Each experimental group contains 11-17 axons with 15-54 mobile tracks observed. 

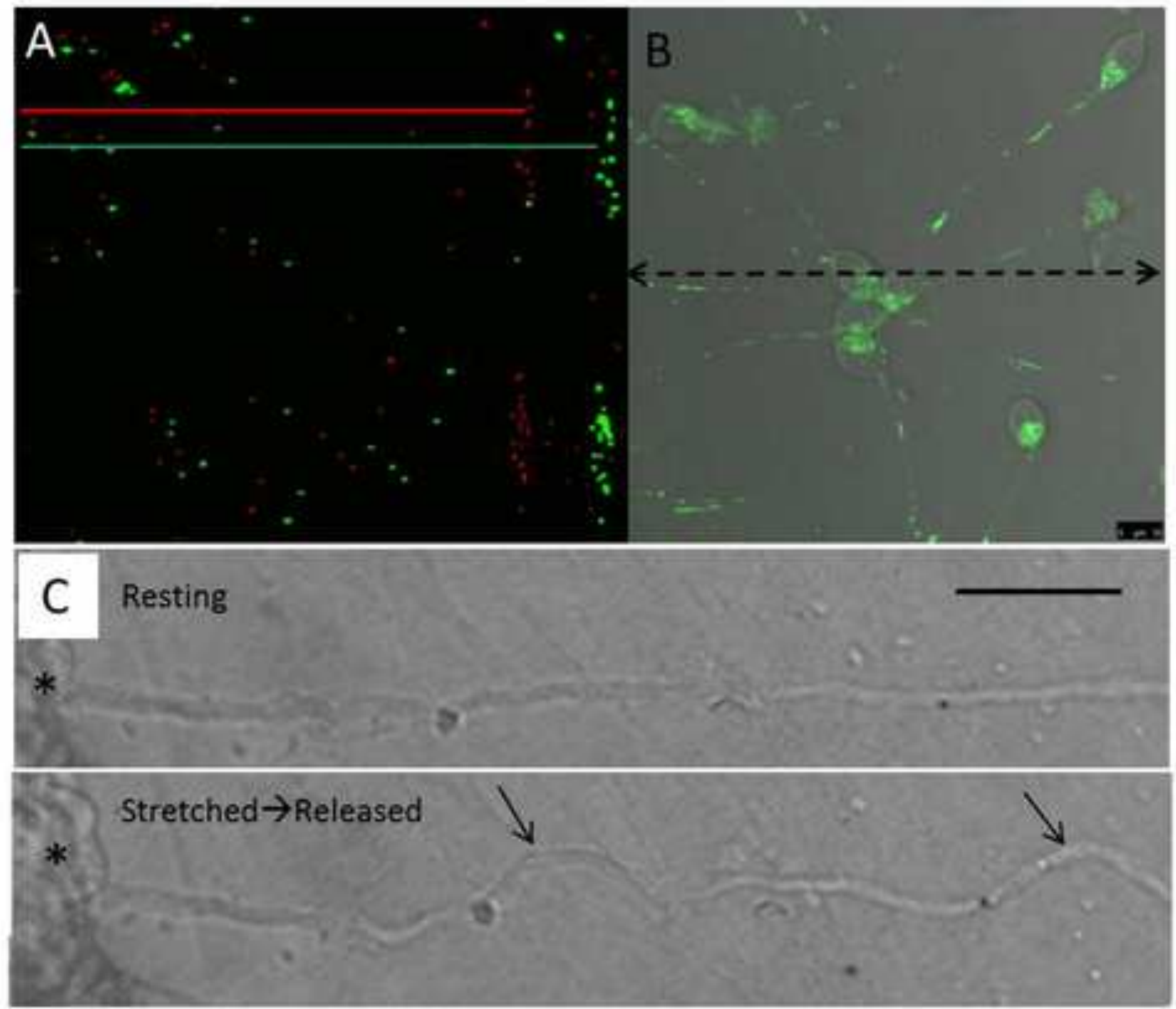

D Resting $y^{f}$

*

Stretched

* 

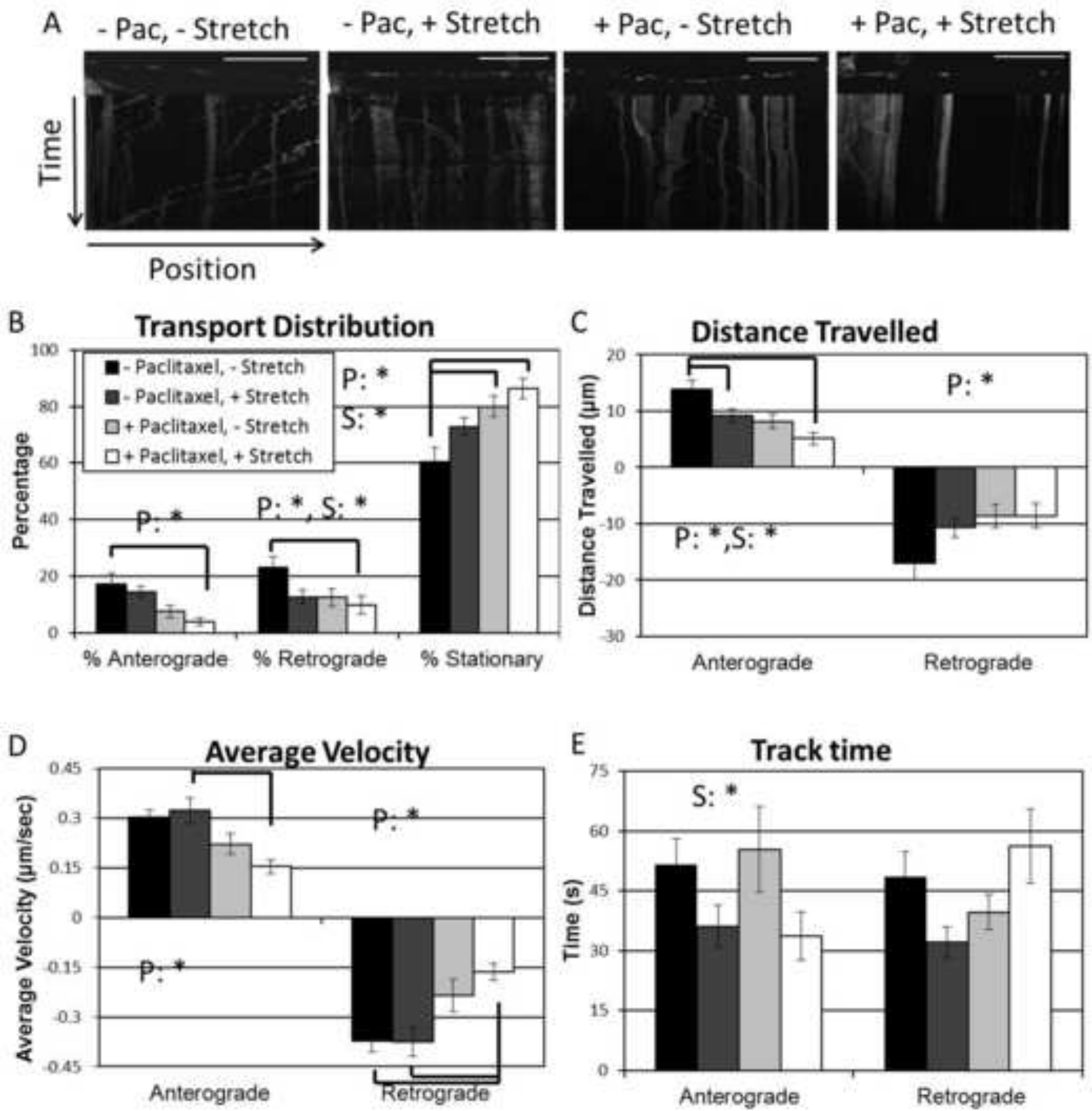

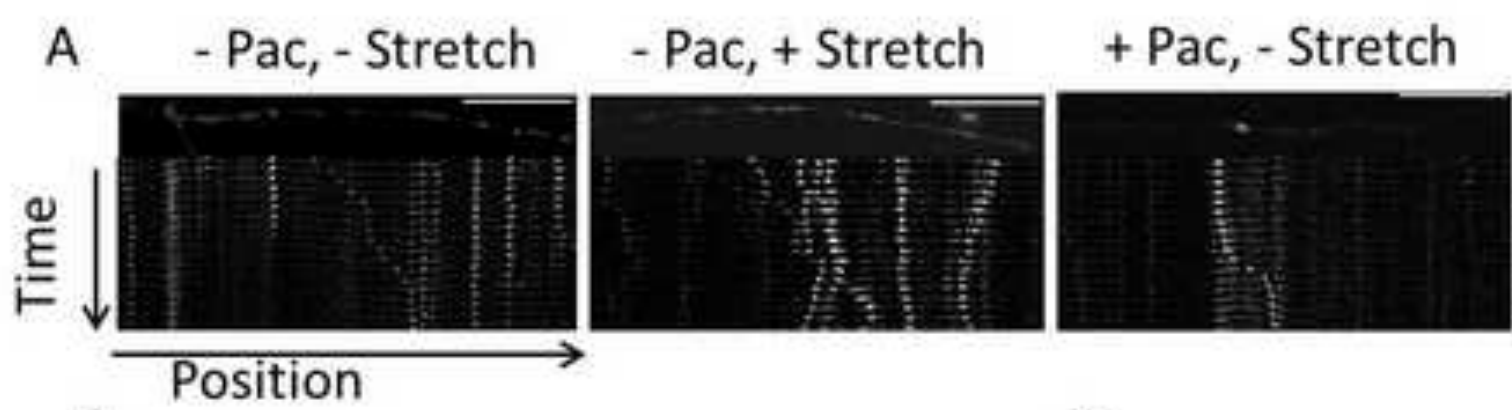

+ Pac, + Stretch

B

Number Mobile Particles

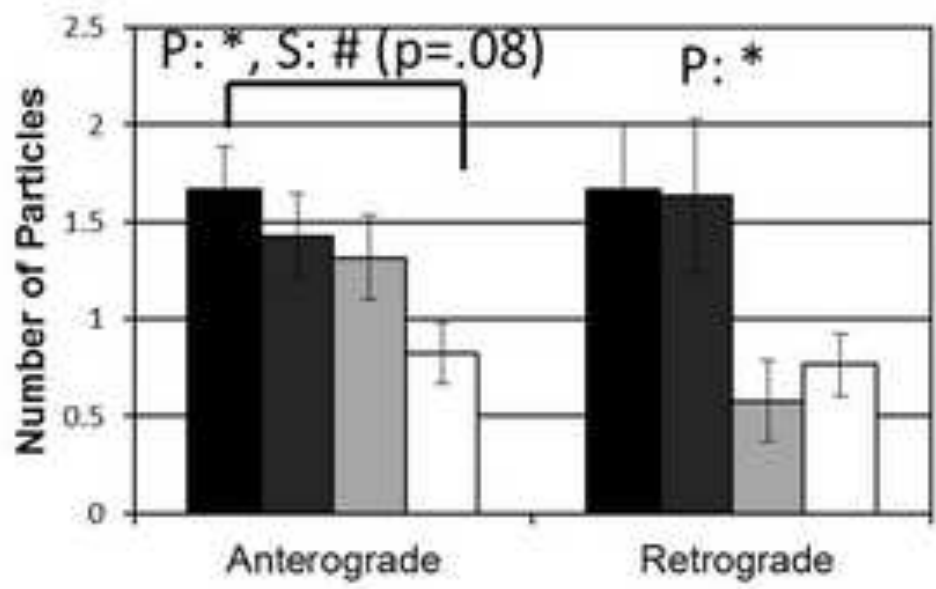

C

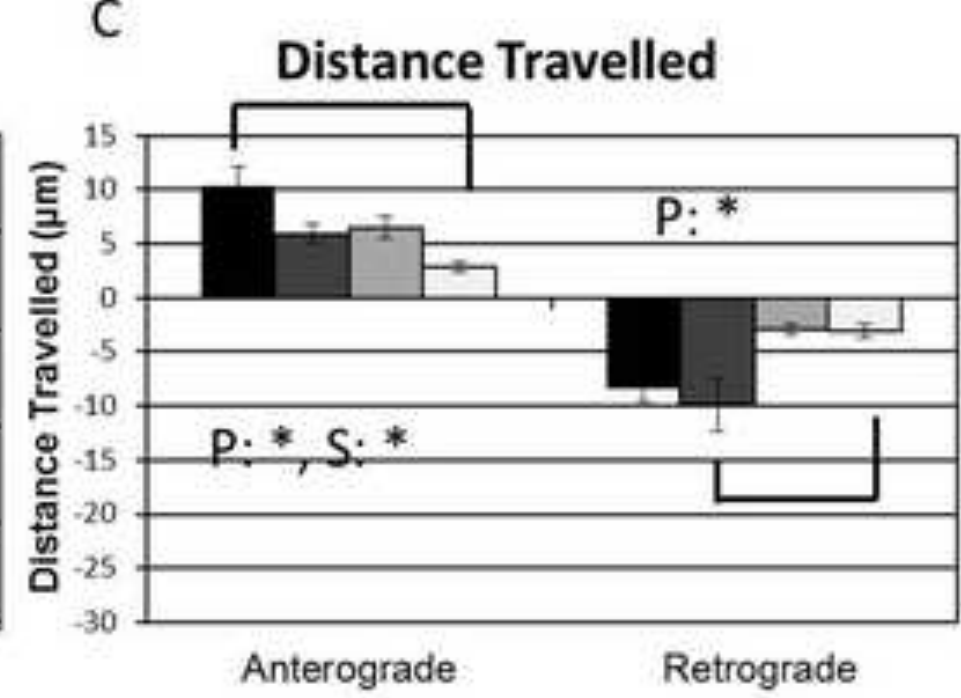

D

Average Velocity

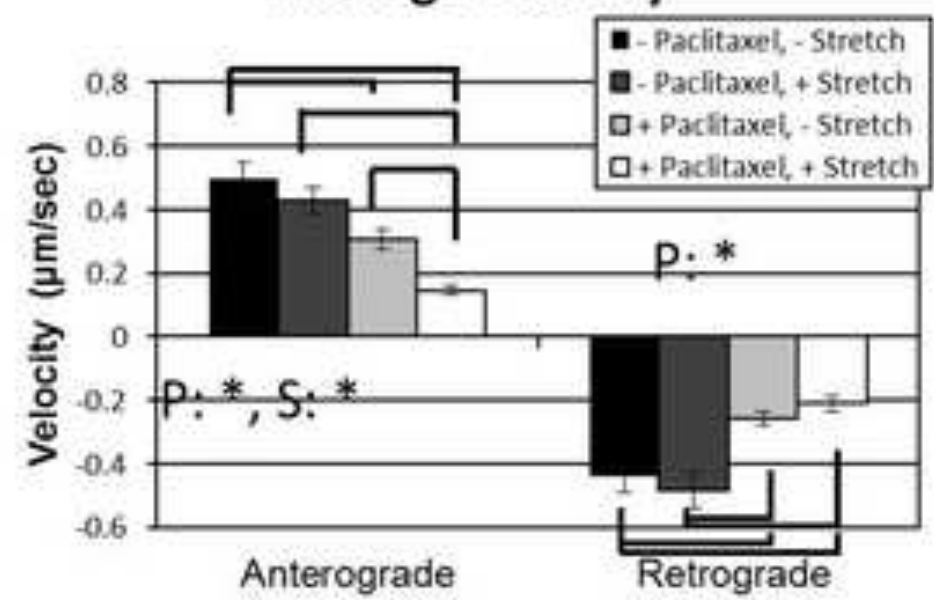

E Track Time (s)

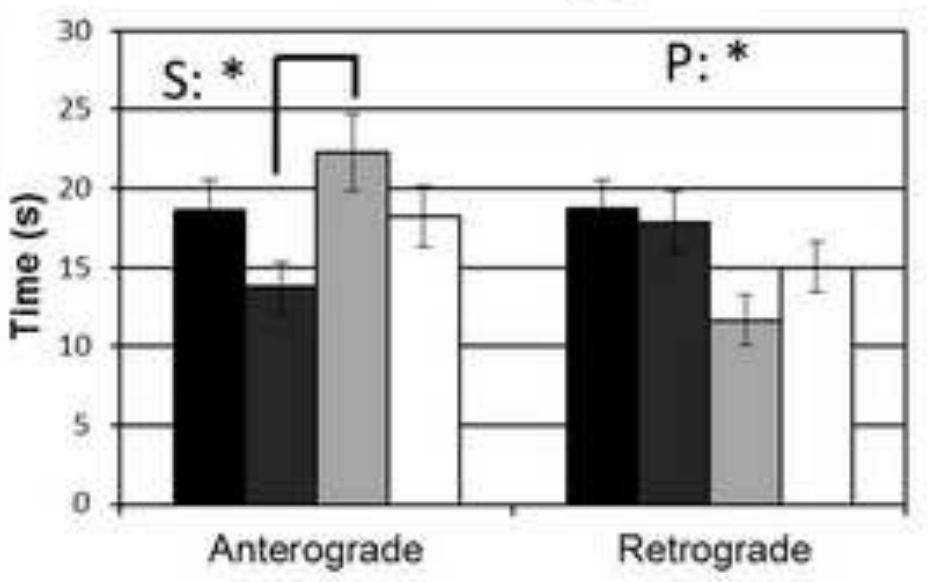




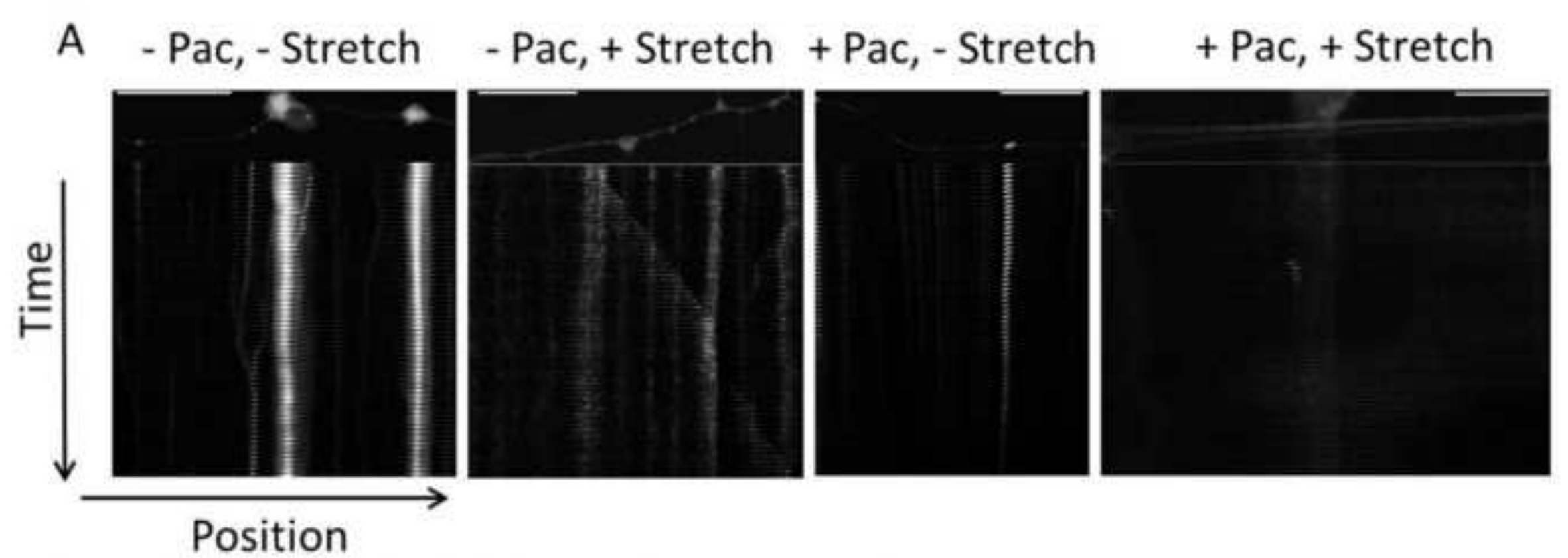

B Number detectable moving

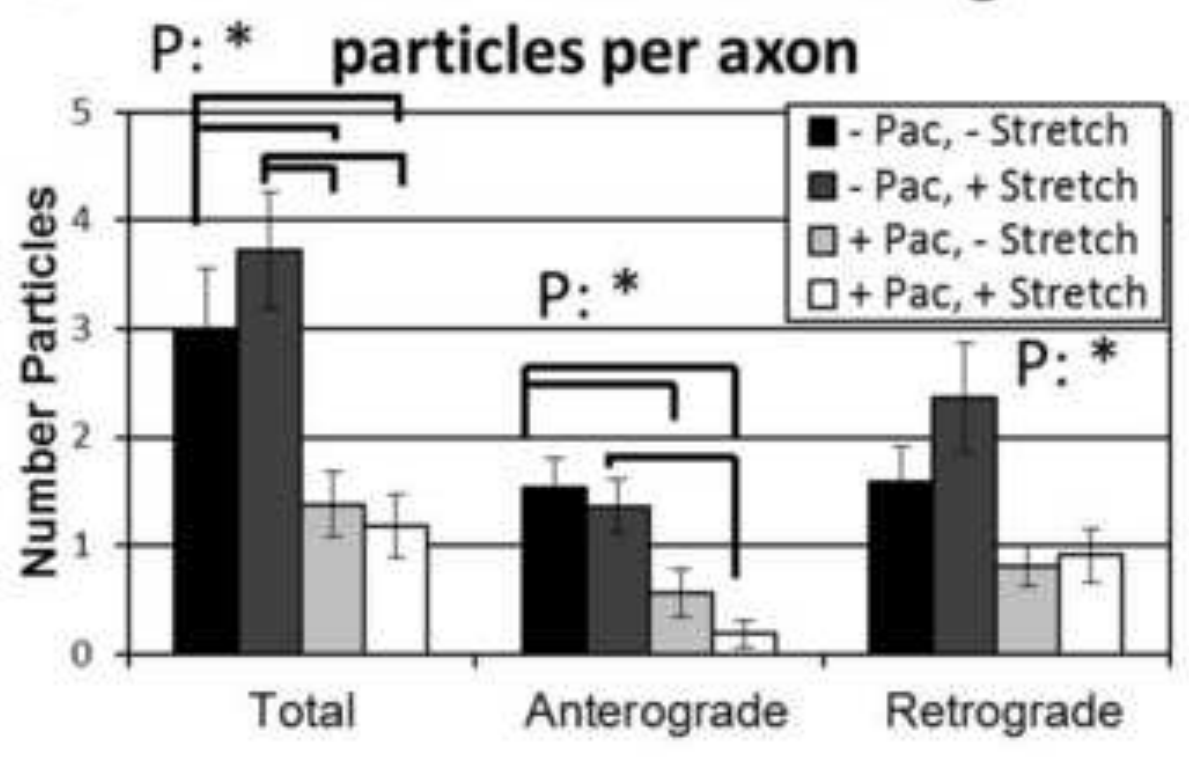

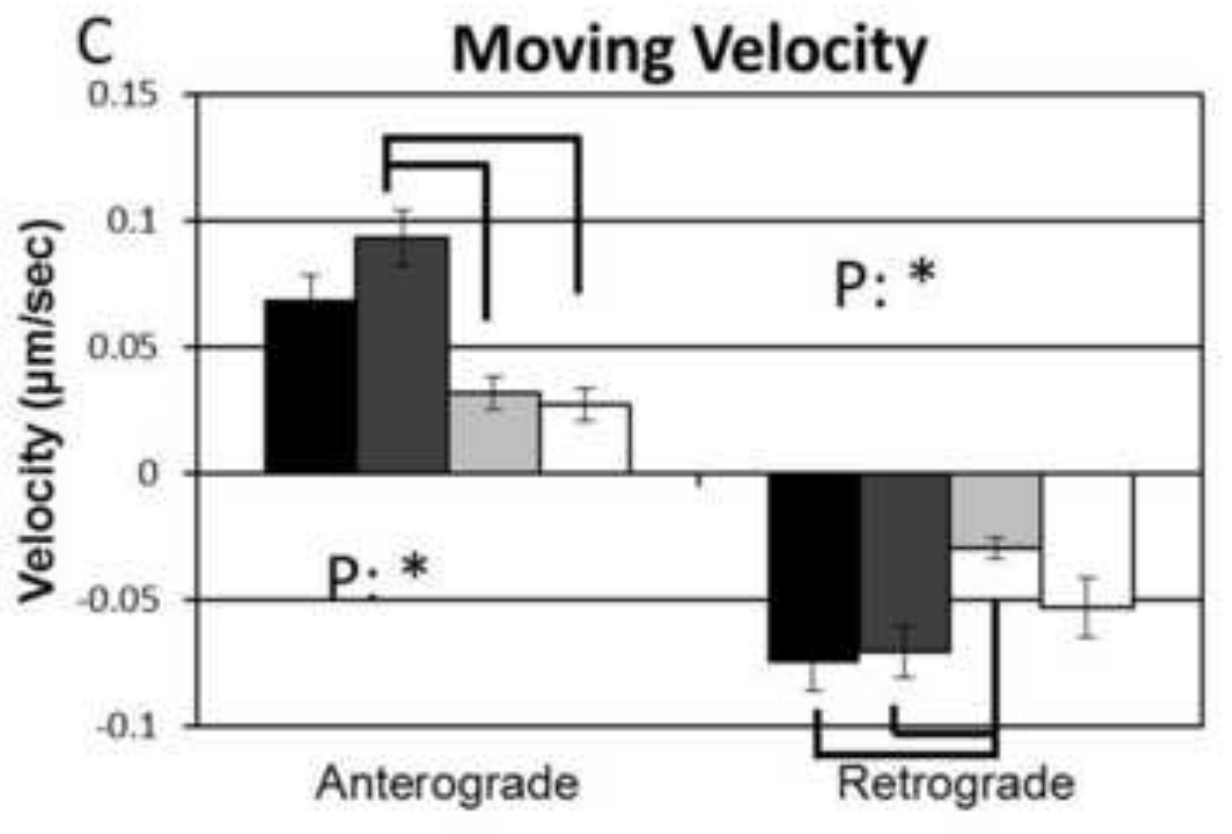

